\title{
Prevalence of methicillin-resistant Staphylococcus aureus colonization in HIV-infected patients in Barcelona, Spain: a cross-sectional study
}

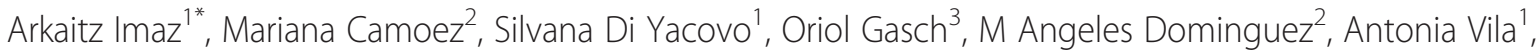 \\ Margarita Maso-Serra' ${ }^{1}$, Miquel Pujol ${ }^{4}$ and Daniel Podzamczer ${ }^{1}$
}

\begin{abstract}
Background: Colonization by community-associated methicillin-resistant Staphylococcus aureus (CA-MRSA) has been found to be markedly more common in HIV-infected individuals in the USA. Studies evaluating the prevalence MRSA colonization in HIV-infected populations in Europe are scarce. The aim of this study was to investigate the prevalence of MRSA colonization in a cohort of HIV-infected patients in Barcelona, Spain.

Methods: Nasal and pharyngeal S. aureus carriage was assessed in a random sample of 190 patients from an outpatient HIV clinic. Nasal and pharyngeal swab specimens were obtained for staphylococcal culture from 190 and 110 patients respectively. All MRSA isolates were screened for Panton-Valentine leukocidin (PVL) genes by PCR. Molecular characterization of MRSA isolates was performed by multilocus sequence typing. Data related to HIV infection, healthcare exposure, and previously described risk factors for MRSA were collected from medical records and a questionnaire administered to each patient.

Results: The patients' characteristics were as follows: male, $83 \%$; median (IQR) age, 45 (39-49) years; intravenous

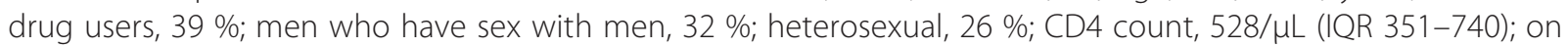
antiretroviral therapy, $96 \%$; and undetectable plasma viral load, $80 \%$. Sixty-five patients (34\%) were colonized by S. aureus. MRSA colonization was found in $1 \%$ and $2 \%$ of nasal and pharyngeal samples respectively. No PVL positive MRSA strains were detected and all the MRSA isolates belonged to typical hospital-acquired clones.
\end{abstract}

Conclusions: Our data suggest that CA-MRSA colonization is not currently a problem in HIV-infected individuals in our area.

Keywords: HIV, Methicillin-resistant Staphylococcus aureus, Colonization

\section{Background}

Methicillin-resistant Staphylococcus aureus (MRSA) has emerged as a causal agent of infection in individuals without risk factors for healthcare- associated MRSA acquisition that has been described as communityassociated MRSA (CA-MRSA) [1]. CA-MRSA infections are usually distinguishable from their healthcare-related counterparts by epidemiological, molecular, and clinical

\footnotetext{
*Correspondence: aimaz@bellvitgehospital.cat

'HIV Unit, Infectious Diseases Department, Hospital Universitari de Bellvitge, L'Hospitalet de Llobregat, Barcelona, Spain

Full list of author information is available at the end of the article
}

features [2]. The incidence of the former is markedly increased in certain population groups such as HIVinfected patients, who have also been noted to have a higher risk of CA-MRSA colonization [3, 4]. In addition, HIV-infected individuals are frequently exposed to healthcare environments, and related factors such as prior hospitalization have also been linked to a higher rate of MRSA colonization in this population [5]. Nasal and extranasal MRSA colonization has been associated with an increased risk of subsequent infections in both HIV-negative and HIV-infected individuals [6, 7]. Additionally, colonization might represent a reservoir for

\section{Ciomed Central}


MRSA transmission. As HIV-infected patients are commonly exposed to healthcare settings, it is important to assess the prevalence of colonization to determine the need for preventive and control measures, at least in hospital settings.

Most studies showing a high prevalence of MRSA colonization among HIV-infected individuals have been conducted in the USA. However, studies in certain US regions [8] and Europe [5, 9-12] have reported low prevalence.

Few European studies have evaluated MRSA colonization or infection in the HIV-infected population [5]. The aim of this study was to assess the prevalence of MRSA colonization in a cohort of HIV-infected patients in Spain.

\section{Methods}

This is a cross-sectional study assessing $S$. aureus colonization in a random sample of 190 adults (>18 years) from a total of 1665 patients seen at an outpatient HIV clinic in Barcelona, Spain between June 2011 and June 2012. Patients were randomly selected using a statistical software package (IBM SPSS version 19.0, Chicago, IL). S. aureus colonization was investigated by taking nasal swabs from all 190 patients and pharyngeal swabs from 110 patients. Swabs were plated onto MRSA agar medium (MRSA Select; Bio-Rad Laboratories, Madrid, Spain) and coagulase-mannitol salt agar plates (BBL ${ }^{\text {ma }}$ Coagulase Mannitol Agar; BD, Madrid, Spain), and also inoculated into staphylococcal enrichment broth (BBL brain-heart infusion; BD, plus $7 \% \mathrm{NaCl}$ ). After $24 \mathrm{~h}$ of incubation at $35-37{ }^{\circ} \mathrm{C}$, the broths were subcultured onto MRSA Select and coagulase-mannitol salt plates. All plates were incubated for $48 \mathrm{~h}$ and inspected daily for S. aureuslike colonies. S. aureus colonies were identified by latex agglutination (Pastorex Staph-plus; Bio-Rad Laboratories) and DNase production (DNase Test Agar; Difco, Fco. Soria Melguizo, Madrid, Spain). Antibiotic susceptibility testing, including cefoxitin, was performed by the disc-diffusion method following CLSI recommendations [13]. All MRSA isolates were screened for Panton-Valentine leukocidin (PVL) genes by PCR. Molecular characterization of MRSA isolates was performed by multilocus sequence typing and SCCmec characterization as described previously $[14,15]$.

HIV infection-related data and epidemiological characteristics previously described as community or healthcarerelated risk factors for MRSA were collected from medical records and a questionnaire completed by each patient (Table 1) [1].

CA-MRSA infection was defined according to the US Centers for Disease Control and Prevention 2000 criteria: 1) Diagnosis of MRSA in an outpatient setting or by a culture positive for MRSA within 48 hours of admission to hospital; 2) No medical history of MRSA infection or colonization; and 3) No medical history in
Table 1 Patient characteristics

\begin{tabular}{|c|c|}
\hline & $(n=190)$ \\
\hline Age, y (median, IQR) & $45(39-49)$ \\
\hline Sex (male/female) & 158/32 (83 \%/17 \%) \\
\hline HIV risk factor & $74(39 \%)$ \\
\hline IDU & $61(32 \%)$ \\
\hline MSM & $49(26 \%)$ \\
\hline Heterosexual & $6(3 \%)$ \\
\hline \multicolumn{2}{|l|}{ Other/unknown } \\
\hline AIDS & $55(29 \%)$ \\
\hline $\mathrm{HCV}$ & $61(32)$ \\
\hline HBV & $8(4 \%)$ \\
\hline Current ART & $182(96 \%)$ \\
\hline $\mathrm{CD}^{+} \mathrm{T}$ cell count, cells/uL (median, IQR) & $528(351-740)$ \\
\hline $\mathrm{CD}^{+} \mathrm{T}$ cell count, $<200$ cells $/ \mu \mathrm{L}$ & 19 (10 \%) \\
\hline HIV RNA, $<40$ copies/mL & $153(80 \%)$ \\
\hline Current use of TMP-SMX & $15(8 \%)$ \\
\hline Origin & Delete \\
\hline Spain & $161(85 \%)$ \\
\hline Europe & $1(0.5 \%)$ \\
\hline South America & $19(10 \%)$ \\
\hline North Africa & $4(2 \%)$ \\
\hline Sub-Saharan Africa & $4(2 \%)$ \\
\hline Asia & $1(0.5 \%)$ \\
\hline \multicolumn{2}{|l|}{ Risk factors for HA-MRSA acquisition } \\
\hline Antibiotic use in prior 12 months & $70(37 \%)$ \\
\hline Hospitalization in prior 12 months & $32(17 \%)$ \\
\hline Intravenous catheter use in prior 12 months & $40(21 \%)$ \\
\hline Surgical intervention in prior 12 months & 19 (10 \%) \\
\hline $\begin{array}{l}\text { Frequent visits ( } \geq 1 \text { per week) to long-term } \\
\text { care facilities }\end{array}$ & 17 (9 \%) \\
\hline Multiple sexual partners in prior 12 months & 17 (9 \%) \\
\hline History of sexually transmitted infection & 17 (9 \%) \\
\hline Previous incarceration & $29(15 \%)$ \\
\hline
\end{tabular}

Data are shown as number (\%) of patients unless otherwise indicated. $I D U$, injecting drug user; IQR, interquartile range; $\mathrm{HBV}$, hepatitis $\mathrm{B}$ virus; $\mathrm{HCV}$, hepatitis C virus; MSM: men who have sex with men;

TMP-SMX: trimethoprim-sulfamethoxazole

the past year of: a) hospitalization; admission to a nursing home, skilled nursing facility, or hospice; c) dialysis; d) surgery; 4) permanent indwelling catheters or medical devices that pass through the skin into the body [1]. Since MRSA clones identified as typically CA-MRSA have distinguishing genetic features from hospital-acquired strains, such as SCCmec types IV and V and PVL expression, molecular characterization was also used to ascertain the possible origin of the strains (community vs healthcare environment) [2]. 
Continuous variables are reported as medians and interquartile range and categorical variables as numbers and percentages. The former were compared using the non-parametric Mann-Whitney U test while the latter were compared using the $\mathrm{X}^{2}$ or Fisher's exact test, with a significance level of 0.05 (two-sided). A multivariate logistic regression model including demographic characteristics (age, gender, origin, HIV acquisition route), CD4+ T cell count, viral load, use of ART, healthcare-related related risk conditions (recent hospitalization or admission to a nursing home, recent surgical intervention, recent use of antibiotics), and other epidemiologic conditions (history of incarceration, history of sexually transmitted infections, multiple sexual partners) was used to identify independent risk factors for $S$. aureus and CA-MRSA colonization.

The study protocol was approved by the Clinical Investigation Ethics Committee of Bellvitge University Hospital and all patients gave written informed consent prior to participation.

\section{Results}

The patients' characteristics are summarized in Table 1. There were no significant statistical differences between patients with nasal and pharyngeal assessment $(n=110)$ and those with nasal assessment only $(n=80)$ (data not shown).

MRSA colonization was detected in 3 patients. Nasal colonization was observed in 2/190 individuals (1\%) and pharyngeal colonization in $2 / 110$ individuals (2\%). Among patients with nasal and pharyngeal samples available, MRSA was present in both nasal and pharyngeal samples in 1 subject whilst the other patient was a pharyngeal carrier exclusively. Among those patients with MRSA colonization, two of them had risk factors for nosocomial acquisition. The two isolates were identified as ST146 and ST125, part of Clonal Complex 5 (CC5), and they both carried SCCmec IV (Table 2), which are indistinguishable features from the dominant hospital-acquired MRSA clone complex in our area [16]. The isolate belonging to the third patient was not available for molecular studies but antibiotic susceptibility and PVL results were available. Although the patient had no apparent risk factors for hospital acquisition, the antibiotic resistance pattern (resistance to erythromycin and ciprofloxacin) was identical to that of the dominant hospital-acquired MRSA lineage (Table 2).

Colonization by methicillin-susceptible $S$. aureus (MSSA) was documented in 62/190 nasal samples (32.6 \%) and 3/ 110 pharyngeal samples (2.7\%), with only 1 in 3 patients found to be an exclusive MSSA pharyngeal carrier. None of the risk factors analyzed, including those related to demography, HIV acquisition route, HIV control, healthcarerelated conditions, and other epidemiologic conditions, was statistically associated with MSSA colonization in either the univariate or multivariate analyses.

\section{Discussion}

The prevalence of S. aureus colonization in our HIVinfected population is consistent with rates reported for the general population [17]. However, only 3 individuals (corresponding to 2/190 nasal samples and 2/110 pharyngeal samples) were colonized by MRSA and none of the isolates belonged to a typical CA-MRSA lineage. Our data contrast with most reports from the USA, where a significant association between HIV infection and MRSA colonization has been well documented [3-7]. A recent meta-analysis assessing MRSA colonization prevalence and risk factors in HIV-infected individuals reported that $6.9 \%$ of the population studied were MRSA carriers and that this rate rose to $8.8 \%$ when only studies from North America were included [5]. Although data on MRSA carriage in HIV-infected individuals in Europe are scarce, lower prevalence rates have been reported (0 \% to $2.8 \%$ ) $[5,9-12]$, and the findings in our series are consistent with reports from other European studies.

Several US studies have described an association between CA-MRSA colonization and risk factors such as sexual behavior, substance abuse, incarceration, and area of residence $[4,6,7]$. These risk groups were represented in our sample (Table 1).

We recently reported a series of MRSA infection in HIV-infected patients in Spain in which we detected a higher risk for MRSA infection among individuals with poorly controlled HIV infection and immigrants (mostly South Americans) [18]. Nevertheless, the overall prevalence was still lower than that reported for the USA. The absence of CA-MRSA colonization in HIV-infected individuals in our area could partly explain the low rate of CA-MRSA infection in this population.

Table 2 Characterization of MRSA isolates

\begin{tabular}{llllll}
\hline Patient & Sample & SCCmec & MLST & PVL & Antibiotic resistance pattern \\
\hline A & Pharyngeal & Type IV & ST146 (CC5) & Negative & Resistance to: erythromycin, clindamycin, tobramycin and ciprofloxacin \\
B & Nasal and Pharyngeal & Type IV & ST125 (CC5) & Negative & Resistance to: erythromycin, tobramycin and ciprofloxacin \\
C & Nasal & NA & NA & Negative & Resistance to: erythromycin, tobramycin and ciprofloxacin \\
\hline
\end{tabular}

CC, clonal complex; MLST, multilocus sequence typing; PVL, Panton-Valentine Leukocidin; SCCmec, staphylococcal cassette chromosome mec; ST, sequence type 
Our study is the first to assess the prevalence of MRSA colonization in HIV-infected patients in Spain. However, it has some limitations. Although our sample is representative of our cohort of HIV-infected patients, the number of individuals studied is lower than in other series. Additionally, while MRSA colonization of extranasal areas such as the buttocks and the perianal, inguinal and axillary regions has been well documented $[4,7,19,20]$, the only extranasal location studied in our case was the pharynx, and paired nasal and pharyngeal samples were only available for a subset of 110 patients. Thus, the rate of MRSA colonization in our cohort might have been underestimated. The importance of extranasal colonization has been demonstrated for both hospital- and communityacquired MRSA $[4,7,19,20]$. Recent studies in the community setting have observed that nasal-only screening could miss up to $51 \%$ of MRSA colonized individuals $[4,19]$. In the subset of patients with both nasal and pharyngeal samples in our series, MRSA was detected in both samples in one patient while the other one was an exclusive pharyngeal carrier. None of the patients had exclusive nasal colonization in this subgroup. Although we do not discount the importance of extranasal colonization (the colonization rate would have been $50 \%$ lower if our study had been limited to nasal samples), the rate of MRSA colonization in our cohort was low.

We were unable to genetically characterize one MRSA isolate. However, PVL expression, which is characteristic in CA-MRSA strains in our area, was not detected, and the antibiotic susceptibility pattern, while unspecific, was indistinguishable from the dominant hospital-acquired MRSA lineage. Thus, the molecular characterization results for the available isolates, the absence of PVL production, and the antibiotic resistance patterns detected suggested healthcare-related acquisition in all cases.

\section{Conclusions}

Although nasal and/or pharyngeal S. aureus colonization was observed in one-third of HIV-infected patients in our cohort, the prevalence of MRSA carriage was low and colonization by typical CA-MRSA strains was not observed. Thus, MRSA colonization in the HIV-infected population in our area does not seem to be an epidemiological problem requiring specific control strategies to prevent cross-transmission and infection among HIVinfected individuals. More studies are needed to assess MRSA colonization rates in different European HIVinfected populations.

\section{Conflict of interests}

The authors declare that they have no competing interests.

\section{Authors' contributions}

$\mathrm{Al}$ and DP initiated this project. OG and MP contributed to the design of the study. Al, MM, AV and DP were responsible for sample collection. MC and MAD performed all microbiological procedures. Al and SDY were responsible for data collection and statistical analysis. Al drafted the manuscript and SDY, MC, MAD, OG, MP and DP reviewed it. All authors revised the manuscript for important intellectual content and contributed to the final version.

\section{Acknowledgments}

This study would not have been possible without the collaboration of all the patients, physicians, and nurses who took part in the project.

We thank Anne Murray for her assistance in the English language reviewing and editing of the manuscript prior to submission.

Sources of financial support: This study was partially funded by the RD12/

0017/0013 project within the Spanish Research, Development and

Innovation Plan and by the Instituto de Salud Carlos III (Subdirección General de Evaluación) and the European Regional Development Fund.

\section{Author details}

${ }^{1}$ HIV Unit, Infectious Diseases Department, Hospital Universitari de Bellvitge, L'Hospitalet de Llobregat, Barcelona, Spain. ${ }^{2}$ Microbiology Department, Hospital Universitari de Bellvitge, L'Hospitalet de Llobregat, Barcelona, Spain. ${ }^{3}$ Infectious Diseases Department, Corporació Sanitària Parc Taulí, Sabadell, Spain. ${ }^{4}$ Infectious Diseases Department, Hospital Universitari de Bellvitge, L'Hospitalet de Llobregat, Barcelona, Spain.

Received: 1 February 2015 Accepted: 19 June 2015

Published online: 26 June 2015

\section{References}

1. Naimi TS, LeDell KH, Como-Sabetti K, et al. Comparison of community- and health care-associated methicillin-resistant Staphylococcus aureus infection. JAMA. 2003;290:2976-84

2. David MZ, Daum RS. Community-associated methicillin-resistant Staphylococcus aureus: epidemiology and clinical consequences of an emerging epidemic. Clin Microbiol Rev. 2010;23:616-87.

3. Hidron Al, Kourbatova EV, Halvosa JS, et al. Risk factors for colonization with methicillin-resistant Staphylococcus aureus (MRSA) in patients admitted to an urban hospital: emergence of community-associated MRSA nasal carriage. Clin Infect Dis. 2005;41:159-66.

4. Popovich KJ, Hota B, Aroutcheva A, et al. Community-associated methicillinresistant Staphylococcus aureus colonization burden in HIV-infected patients. Clin Infect Dis. 2013;56:1067-74.

5. Zervou FN, Zacharioudakis IM, Ziakas PD, Rich JD, Mylonakis E. Prevalence of and risk factors for methicillin-resistant Staphylococcus aureus colonization in HIV infection: a meta-analysis. E Clin Infect Dis. 2014;59:1302-11.

6. Shet A, Mathema B, Mediavilla JR, et al. Colonization and subsequent skin and soft tissue infection due to methicillin-resistant Staphylococcus aureus in a cohort of otherwise healthy adults infected with HIV type 1. J Infect Dis. 2009;200:88-93.

7. Szumowski JD, Wener KM, Gold HS, et al. Methicillin-resistant Staphylococcus aureus colonization, behavioral risk factors, and skin and soft-tissue infection at an ambulatory clinic serving a large population of HIV-infected men who have sex with men. Clin Infect Dis. 2009;49:118-21.

8. Madariaga MG, Ullrich F, Swindells S. Low prevalence of communityacquired methicillin-resistant Staphylococcus aureus colonization and apparent lack of correlation with sexual behavior among HIV-infected patients in Nebraska. Clin Infect Dis. 2009;48:1485-7.

9. Giuliani M, Longo B, Latini A, et al. No evidence of colonization with community-acquired methicillin-resistant Staphylococcus aureus in HIV-1infected men who have sex with men. Epidemiol Infect. 2010;138:738-42.

10. Oliva A, Lichtner M, Mascellino MT, et al. Study of methicillin-resistant Staphylococcus aureus (MRSA) carriage in a population of HIV-negative migrants and HIV-infected patients attending an outpatient clinic in Rome. Ann lg. 2013;25:99-107.

11. Seybold U, Supthut-Schroder B, Draenert R, Hogardt M, Bogner JR. Prevalence and risk factors of nasal colonization with Staphylococcus aureus - association with HIV infection in older patients. Scand J Infect Dis. 2009;41:63-6.

12. Joore IK, van Rooijen MS, Schim van der Loeff MF, de Neeling AJ, van Dam A, de Vries HJ. Low prevalence of methicillin-resistant Staphylococcus aureus among men who have sex with men attending an STI clinic in Amsterdam: a cross-sectional study. BMJ Open. 2013;3(3):pii:e002505.

13. CLSI (2010) Performance standards for antimicrobial susceptibility testing; 20th Informational Supplement. CLSI document M100-S20. Wayne, pa: Clinical and laboratory standards institute; 2010. 
14. Enright MC, Day NP, Davies CE, Peacock SJ, Spratt BG. Multilocus sequence typing for characterization of methicillin-resistant and methicillin-susceptible clones of Staphylococcus aureus. J Clin Microbiol. 2000;38(3):1008-15.

15. Oliveira DC, De Lencastre H. Multiplex PCR strategy for rapid identification of structural types and variants of the mec element in methicillin-resistant Staphylococcus aureus. Antimicrob Agents Chemother. 2002;46:2155-61.

16. Gasch O, Camoez M, Dominguez MA, et al. Predictive factors for mortality in patients with methicillin-resistant Staphylococcus aureus bloodstream infection: impact on outcome of host, microorganism and therapy. Clin Microbiol Infect. 2013;19(11):1049-57.

17. Schechter-Perkins EM, Mitchell PM, Murray KA, Rubin-Smith JE, Weir S, Gupta K. Prevalence and predictors of nasal and extranasal staphylococcal colonization in patients presenting to the emergency department. Ann Emerg Med. 2011:57:492-9.

18. Imaz A, Cobos-Trigueros N, Falcó V. Community-associated methicillinresistant Staphylococcus aureus infections in HIV-infected patients in Spain. J Infect. 2013;66:199-201.

19. Miller LG, Eells SJ, Taylor AR, et al. Staphylococcus aureus colonization among household contacts of patients with skin infections: risk factors, strain discordance, and complex ecology. Clin Infect Dis. 2012;54:1523-35.

20. McKinnell JA, Huang SS, Eells SJ, Cui E, Miller LG. Quantifying the impact of extranasal testing of body sites for methicillin-resistant Staphylococcus aureus colonization at the time of hospital or intensive care unit admission. Infect Control Hosp Epidemiol. 2013;34:161-70.

\section{Submit your next manuscript to BioMed Central and take full advantage of:}

- Convenient online submission

- Thorough peer review

- No space constraints or color figure charges

- Immediate publication on acceptance

- Inclusion in PubMed, CAS, Scopus and Google Scholar

- Research which is freely available for redistribution 